\title{
The Changes in the Immunocytochemical Localization of Cathepsin $L$ and Type I Collagen in Rat Osteoclasts Treated with E-64
}

\author{
Ryoji Moroi', Takayoshi Yamaza², Yasunori Ayukawa ${ }^{3}$, Tamotsu Kiyoshima ${ }^{5}$, \\ Yasuyoshi Ohsaki ${ }^{4}$, Yukio Nishimura ${ }^{6}$, Yoshihiro Terada $^{1}$, \\ Masaru Himeno ${ }^{6}$ and Teruo Tanaka ${ }^{2}$ \\ Department of Prosthetic Dentistry I', II', Oral Anatomy $I^{2}, I^{4}$, and Oral Pathologys, Faculty of Dentistry, Kyushu \\ University and Department of Physiological Chemistry ${ }^{6}$, Faculty of Pharmaceutical Science, Kyushu University, \\ Fukuoka, 812-82
}

Received for publication January 22, 1996

\begin{abstract}
The localization of cathepsin L or type I collagen in the osteoclasts (rat femur) treated with or without E-64 (control) was examined immunocytochemically to investigate how E64 affects the osteoclasts. Using a light microscope in the E-64-treated osteoclasts, the immunoreactivity for cathepsin $L$ was extracellularly very weak compared with that in the control osteoclasts, but was strong intracellularly. The intracellular immunoreactivity for type I collagen was found in the vacuoles in the E-64-treated osteoclasts but not in any vacuoles in the control osteoclasts. On the other hand, the extracellular immunoreactivity along the resorption lacunae of the E-64-treated osteoclasts was somewhat weaker than that of the control osteoclasts. Using electron microscopy in the E-64-treated osteoclasts, only a small

number of extracellular immunoreaction products for cathepsin $\mathrm{L}$ were seen along the resorption lacunae. In addition, intracellular cathepsin $L$ was deposited in the endosomelysosomal vacuoles which were welldeveloped by E-64. Gold particles indicating type I collagen appeared on the bone matrix, and they were also detected in the vacuoles and vesicles in the E-64-treated osteoclasts. However, they were not detected in the organelles of the control osteoclasts. Thus, in the E-64-treated osteoclasts, the extracellular release of cathepsin $L$ was insufficient to suppress the degradation of collagen. In addition, the undegraded collagen seemed to be endocytosed. The above findings thus suggest that bone resorption is inhibited by this incomplete degradation of collagen.
\end{abstract}

Key words: Osteoclasts, E-64, Cathepsin L, Type I collagen, Immunocytochemistry

\section{Introduction}

Osteoclasts are the cells that remove the bone mineral and degrade the bone organic matrix [4]. The bone surface under the osteoclast ruffled border is thought to be demineralized by hydrogen ions secreted by proton pump located at the ruffled border $[2,34]$, and the bone organic matrix is considered to be degraded by such proteinases as cysteine proteinases and/or collagenase, released by osteoclasts $[5,8,10,12,14,15,19]$. Cathepsins $B, L$ and $\mathrm{N}$ have been shown to degrade collagen at an acidic $\mathrm{pH}$, and a comparison of collagenolytic activity of these pro-

Correspondence to: Prof. Teruo Tanaka, Department of Oral Anatomy I, Faculty of Dentistry, Kyushu University, 3-1-1 Maidashi, Higashi-ku, Fukuoka, 812-82, Japan. teinases showed that cathepsin $\mathbf{L}$ was particularly strong $[22,30]$. The immunocytochemical localization of cathepsins $B, D$ and $L$ in an eroded bone surface under osteoclastic resorption lacunae was examined by Goto et al. [13-15] and cathepsin $L$ was thus found to be much stronger than other cathepsins. The binding affinity of cathepsin $L$ for collagen is much higher than cathepsins $B$, $\mathrm{N}$, and S [25]. Ngyuen et al. [27] reported that cartilage proteoglycan is degraded more extensively by cathepsin $\mathrm{L}$ than by cathepsin B. Therefore, cathepsin L is considered to play an important role in the degradation of the bone organic matrix by osteoclasts.

E-64 is a powerful inhibitor of all kinds of cysteine proteinases [16, 18]. Although Delaissé et al. $[6,7]$ and Kakegawa et al. [21] reported that E-64 reduces osteoclastic bone resorption, these reports have all been 
restricted to biochemical data. Recently, Debari et al. [3] demonstrated the effect of cysteine proteinase inhibitors morphologically by using an ultrastructural assay system. However, they only examined the resorption lacunae on dentine slices but not the osteoclasts themselves.

Type I collagen is a most popular organic matrix in bone. In normal osteoclasts, this collagen seems to be degraded in the resorption lacunae (extracellular spaces) under proteinase enzymes, and therefore is not seen in any vacuoles of the osteoclasts. On the other hand, Everts et al. $[10,11]$ reported the presence of collagen fibrils in the vacuoles of osteoclasts treated with either leupeptin or E64. This phenomenon may result from an insufficient degradation of collagen fibrils due to the inhibition of cysteine proteinases by inhibitors. However, only 4\% of the osteoclasts under the influence of such proteinase inhibitors exhibited the presence of collagen in the vacuoles. It is therefore very difficult to directly observe collagen fibrils in vacuoles. An immunocytochemical method using a specific antibody of type I collagen may thus make the observation of collagen in the vacuoles easier.

The present study was designed to immunocytochemically examine the localization of cathepsin $L$ and type I collagen in rat osteoclasts treated either with or without E-64 in vivo using the antibody for cathepsin L or type I collagen in order to investigate how E-64 affects the osteoclasts.

\section{Materials and Methods}

Four-week old male Wistar rats $(60-70 \mathrm{~g})$ were injected intraperitoneally with physiological saline supplemented E-64 (1, 2, 4 or $8 \mathrm{mg} / 100 \mathrm{~g}$ body weight). A total 36 rats were used in all (9 rats for each dose of E-64). In addition, 6 control rats (same age males) were injected with only physiological saline. The optimal duration $(6 \mathrm{hr})$ for the use of E-64 and the optimal dosage of E-64 were determined based on the data of previous studis [6, 17]. The rats were anesthetized by ether and pentobarbital sodium $6 \mathrm{hr}$ after the injection of E-64 or only saline. Thereafter they were perfused with $0.05 \%$ glutaraldehyde, $4 \%$ paraformaldehyde and $0.2 \%$ picric acid in $0.1 \mathrm{M}$ phosphate buffer $(\mathrm{PB})$ at $4^{\circ} \mathrm{C}$, and the femoral proximal growth plates were dissected from the rats, and further fixed with the same fixative for $3 \mathrm{hr}$ at $4^{\circ} \mathrm{C}$. After fixation, the specimens were washed with $\mathrm{PB}$ and decalcified in 5\% EDTA containing $4 \%$ sucrose for 6 days at $4^{\circ} \mathrm{C}$.

\section{Immuno-light microscopy}

The procedures used for the observation at the light microscopic level of the localization of the cathepsin $L$ or type I collagen in the bone were almost the same as those previously reported by Goto et al. [13-15]. The specimens from the rats with E-64 or only saline were cytoprotected by incubation overnight at $4^{\circ} \mathrm{C}$, with $2.3 \mathrm{M}$ sucrose in $\mathrm{PB}$, and frozen in liquid nitrogen. The frozen specimens were cut into semi-thin sections measuring $1 \mu \mathrm{m}$ in thickness on the Richert Ultracut $\mathrm{E}$ equipped with an FC4E cryoattachment with glass knives at $-70^{\circ} \mathrm{C}$, and placed on gelatincoated slides. The sections were washed with $0.1 \mathrm{M}$ phosphate-buffered saline (PBS, pH 7.2) and stained immunohistochemically at room temperature by the avidinbiotin-peroxidase complex (ABC) method with a Vectastein ABC kit (Vector Laboratories, Bulingame, Calif., USA).

Firstly the sections were treated with $0.3 \% \mathrm{H}_{2} \mathrm{O}_{2}$ in PBS for $1 \mathrm{hr}$ to inhibit endogenous peroxidase, and subsequently were placed in a humidity chamber. The sections were washed with PBS and preincubated for $15 \mathrm{~min}$ with 3\% normal goat serum in PBS to block the non-specific sites. The sections were then incubated with (1) the primary antibodies for $1.5 \mathrm{hr}$; (2) biotinylated goat antirabbit IgG diluted $1: 200$ in PBS for $30 \mathrm{~min}$; and (3) ABC complex for $45 \mathrm{~min}$. After washing with PBS, the sections were reacted with diaminobenzidine tetrahydrochloride (Dojindo Laboratories, Kumamoto, Japan) and $0.01 \mathrm{ml}$ of $33 \% \mathrm{H}_{2} \mathrm{O}_{2}$ in $50 \mathrm{ml}$ of $0.05 \mathrm{M}$ TRIS- $\mathrm{HCl}$ buffer (pH 7.6) for 3-5 min, and were then counterstained with toluidine blue.

Immunohistochemical controls using part of the above sections were performed as follows: 1) the primary antibody was either omitted or replaced by a non-immune IgG: 2) the primary antiserum was substituted for the serum preabsorbed with an excess of either cathepsin $L$ or type I collagen.

\section{Immuno-electron microscopy}

For the pre-embedding method, the specimens from rats with E-64 or only saline were first cut into sections measuring $100 \mu \mathrm{m}$ in thickness with a Microslicer (DTK 1000, Dosaka EM; Kyoto, Japan). The sections were immunocytochemically stained by the $\mathrm{ABC}$ method at $4^{\circ} \mathrm{C}$. They were treated with $0.3 \% \mathrm{H}_{2} \mathrm{O}_{2}$ in $\mathrm{PBS}$ for $1 \mathrm{hr}$ to block endogenous peroxidase. According to the method of Baron et al. [1], they were incubated with primary antibody for cathepsin L overnight, followed by washing with PBS and incubation overnight with the anti-rabbit biotinylated antibody diluted $1: 200$ with PBS. After being washed with PBS, as described above, the sections were immunostained overnight with $\mathrm{ABC}$ reagent and then were incubated with $\mathrm{DAB}$ solution for $10 \mathrm{~min}$ at room temperature. After washing of the sections with PBS, they were postfixed in $0.1 \% \mathrm{OsO}_{4}$ for $1 \mathrm{hr}$ at room temperature, and then dehydrated in a graded series of ethanol and embedded in Quetol 653. The ultrathin sections that were cut and mounted on copper grids were not counterstained and then were examined by JEOL (Tokyo, Japan) JEM1210 electron microscopy.

In the post-embedding method, the sections measuring $100 \mu \mathrm{m}$ in thickness were dehydrated in a graded series of ethanol at a low temperature, and embedded in LR Gold Resin (The London Resin Co. Ltd., Working, UK). 
The polymerization of LR Gold Resin by U.V. irradiation (360 $\mathrm{nm}$ peak wavelength) was done at from -20 to $-30^{\circ} \mathrm{C}$ in a freezer for $24 \mathrm{hr}$. Ultrathin sections embedded in LR Gold Resin were mounted on 200 mesh nickel grids coated with formvar. The grids were blocked with 3\% normal goat serum in $0.1 \mathrm{M}$ PBS for $10 \mathrm{~min}$ at room temperature and then were incubated with the primary antibody for type I collagen for $2 \mathrm{hr}$ at room temperature. After washing with $0.1 \mathrm{M}$ PBS, the grids were incubated with colloidal gold-labeled goat anti-rabbit IgG (gold particles $15 \mathrm{~nm}$ in diameter, $1: 100$ dilution) for $30 \mathrm{~min}$ at room temperature. The grids were counterstained with $2 \%$ uranyl acetate and then were examined with JEM1210 electron microscopy.

\section{Antibodies}

The antibody against cathepsin $\mathrm{L}$ from rat kidney and antibody against type I collagen from mouse skin were raised in rabbits as previously described [28, 31]. In addition, the antibody against mouse type I collagen was confirmed to crossreact with rat type I collagen by Western blot.

\section{Results}

Before immunostaining for rat osteoclasts, we morphologically examined the effects of E-64 for osteoclasts. In the osteoclasts treated with $8 \mathrm{mg} / 100 \mathrm{~g} \mathrm{~b} . w$. of E-64, based on the effects of an overadministration of E-64, numerous huge vacuoles appeared in the cytoplasm, and the rupture of their ruffled borders was found most frequently (Fig. 1). Thus, the overadministration of E-64 showed the osteoclasts to have a cytotoxic effect. On the other hand, in the osteoclasts treated with $1 \mathrm{mg} / 100 \mathrm{~g}$ b.w. of E-64, although the endosome-lysosomal vacuoles were observed to be well-developed, the other morphological findings were very similar to those of the control osteoclasts. Thus, the osteoclasts treated with $1 \mathrm{mg} \mathrm{E-64}$ were immunohistochemically compared with the control osteoclasts without E-64.

\section{Immuno-light microscopy}

The control osteoclasts or E-64 (1 mg)-treated osteoclasts in the cryosections were stained with the ABC method using either anti-cathepsin $L$ or anti-type $I$ collagen. In the localization of cathepsin $L$ in the control osteoclasts, a strong extracellular immunoreactivity was noted along the resorption lacunae. In contrast only a weak intracellular immunoreactivity was observed in a few vacuoles and vesicles (Fig. 2), as previously described by Goto et al. $[14,15]$. On the other hand, in the E-64treated osteoclasts, the intracellular immunoreactivity for capthepsin $\mathbf{L}$ was found in such organelles as vesicles, granules and vacuoles (Fig. 3), and these cathepsin L-positive organelles were both more numerous and demonstrated a stronger immunoreactivity than those in the control osteoclasts. On the other hand, the extracellular immunoreactivity of cathepsin $\mathrm{L}$ was very weak

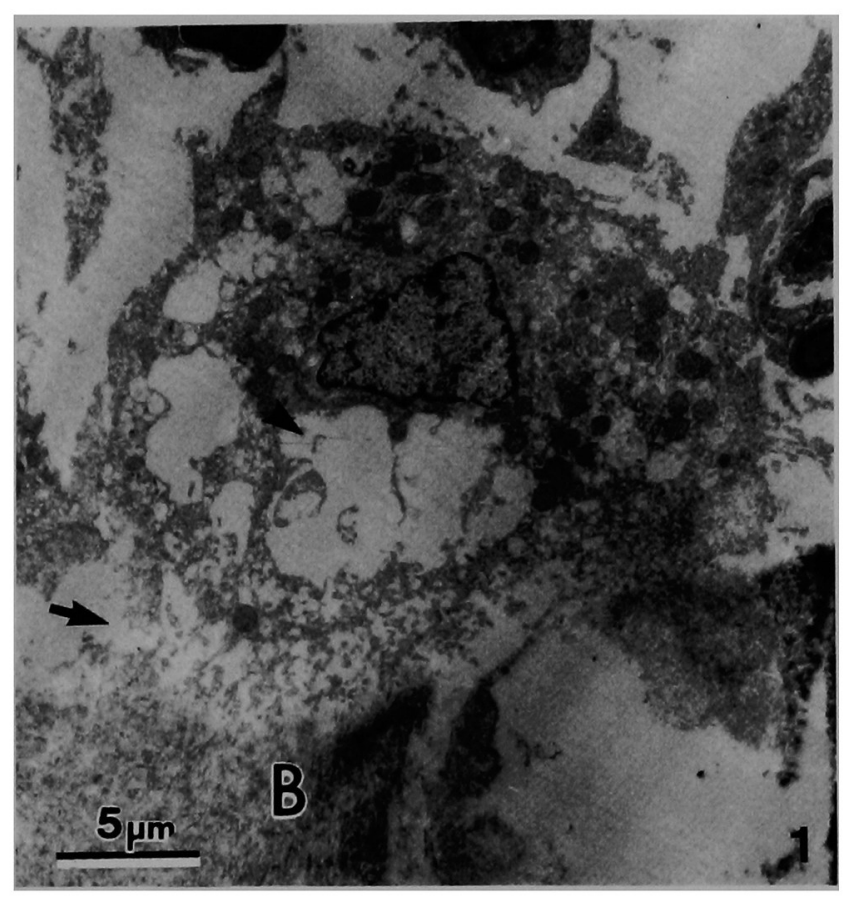

Fig. 1. An electron micrograph of an E-64 (8 mg)-treated osteoclast. Numerous huge vacuoles (arrowhead) and rupturing ruffled borders (arrow) are observed as abnormal changes. Bone (B). Uranyl acetate staining. $\times 3,000$.

along the resorption lacunae (Fig. 3).

The extracellular immunoreactivity for type I collagen along the resorption lacunae facing the E-64-treated osteoclasts was almost the same or only slightly weaker than that along the resorption lacunae facing the control osteoclasts (Figs. 4, 5). The intracellular immunoreactivity for type I collagen was weak, but found in the vacuoles in the E-64-treated osteoclasts (Fig. 5). On the other hand, in the control osteoclasts, no intracellular immunoreactivity was found in any of the vacuoles (Fig. 4).

\section{Immuno-electron microscopy}

The Quetol pre-embedding sections of the control or E-64 (1 mg)-treated osteoclasts were stained with the ABC method using anti-cathepsin L (Figs. 6-8). In the control osteoclasts, the immunoreaction products of cathepsin $\mathrm{L}$ were extracellularly and exclusively deposited along the resorption lacunae under the ruffled borders, on the external surface of the cytoplasmic processes of the ruffled borders, and at the luminal side of the ampullar dilations at the base of the ruffled borders, which appear in the sections as vacuoles. These products were continuous from the luminal side of the ampullar dilations to the surface of the resorption lacunae. Immunoreactivity was also detected in numerous vesicles and granules close to the ruffled border (Fig. 6). In addition, these vesicles sometimes protruded into the ampullar vacuoles at the budding sites of the transport vesicles as previously reported by Baron et al. [1] and Goto et al. [15]. On the other hand, 


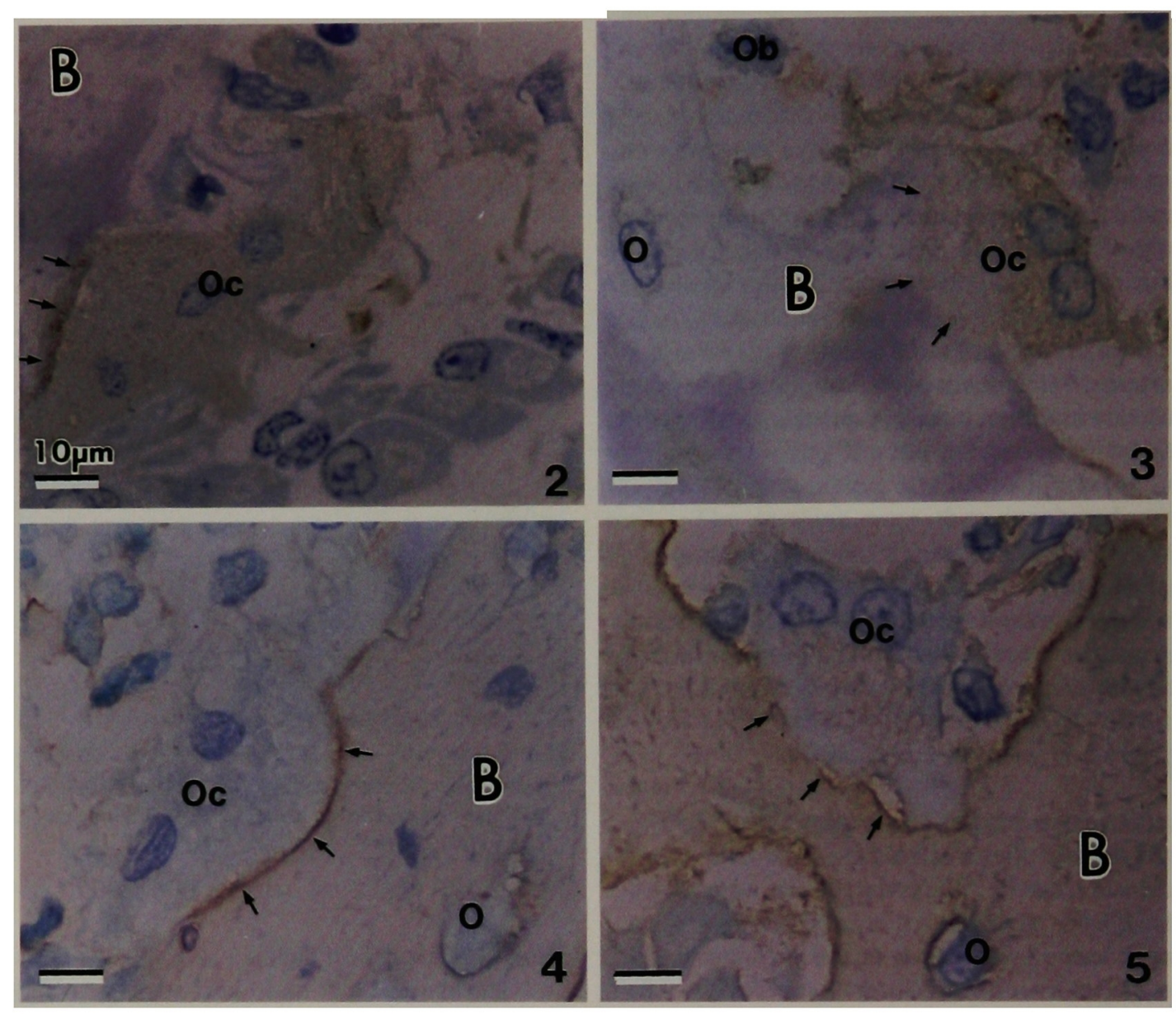

Figs. $2 \& 3$. Light micrographs of $1 \mu$ m-cryostat sections of the control (Fig. 2) or E-64 (1 mg)-treated (Fig. 3) rat femur immunostained with the $\mathrm{ABC}$ method with anti-cathepsin L. In Fig. 2, a strong extracellular immunoreactivity of cathepsin $\mathrm{L}$ is found along the resorption lacuna (arrows), while, in contrast, the intracellular immunoreactivity is weak. In Fig. 3, extracellular immunoreactivity is very weak (arrows), but the intracellular immunoreactivity is strong compared to that in the control osteoclast (Fig. 2) and appears as many granular, vesicular and vacuolar structures. Bone $(B)$, osteoclast $(O c)$, osteocyte $(O)$, osteoblast $(O b)$. Toluidine blue staining. $\times 800$.

Figs. 4 \& 5. Light micrographs of $1 \mu \mathrm{m}$-cryostat sections of the control (Fig. 4) or E-64 (1 mg)-treated (Fig. 5) rat femur immunostained with the $\mathrm{ABC}$ method using anti-type I collagen. In Fig. 4, very strong immuno-reactivity of type I collagen is found along the resorption lacuna (arrows), but no intracellular immunoreactivity appears. In Fig. 5, the extracellular immunoreactivity is discontinuously found along the resorption lacuna of the E-64-treated osteoclast (arrows), and weak intracellular immunoreactivity appears in numerous vacuoles. Bone (B), osteoclast $(O c)$, ostocyte $(O)$. Toluidine blue staining. $\times 800$.

in the E-64-treated osteoclasts, intracellular immunoreactive products were detected in the endosome-lysosomal vacuoles (system) which were well-developed by E-64, whereas the extracellular deposition of cathepsin $\mathrm{L}$ was less than that of cathepsin $\mathrm{L}$ in the control osteoclasts.

The LR Gold resin post-embedding sections of the control or E-64-treated osteoclasts were labeled with the immunogold method using anti-type I collagen (Figs. 911). Numerous colloidal gold particles indicating type I collagen appeared on the bone matrix under the ruffled border of both the control and E-64-treated osteoclasts (Figs. 9-11). There were no differences in the distribution and number of the colloidal golds between both the bone matrix of the control and the E-64-treated osteoclasts. Some banded collagen fibrils under the ruffled borders were labeled with numerous colloidal particles (Fig. 10). In the control osteoclasts, no gold particles were detected between the cytoplasmic processes of the ruffled borders and either in the ampullar or round vacuoles and vesicles close to the ruffled borders (Fig. 9). On the other hand, in the E-64-treated osteoclasts, gold particles indicating type I collagen were detected between the cytoplasmic processes of the ruffled borders, in the large vacuoles, small vacuoles (probably endosomes) and vesicles (probably coated vesicles) close to the ruffled borders, and in the vesicles (lysosomes) close to the nucleus (Fig. 11).

\section{Discussion}

Hashida et al. [17] reported that the concentration of 


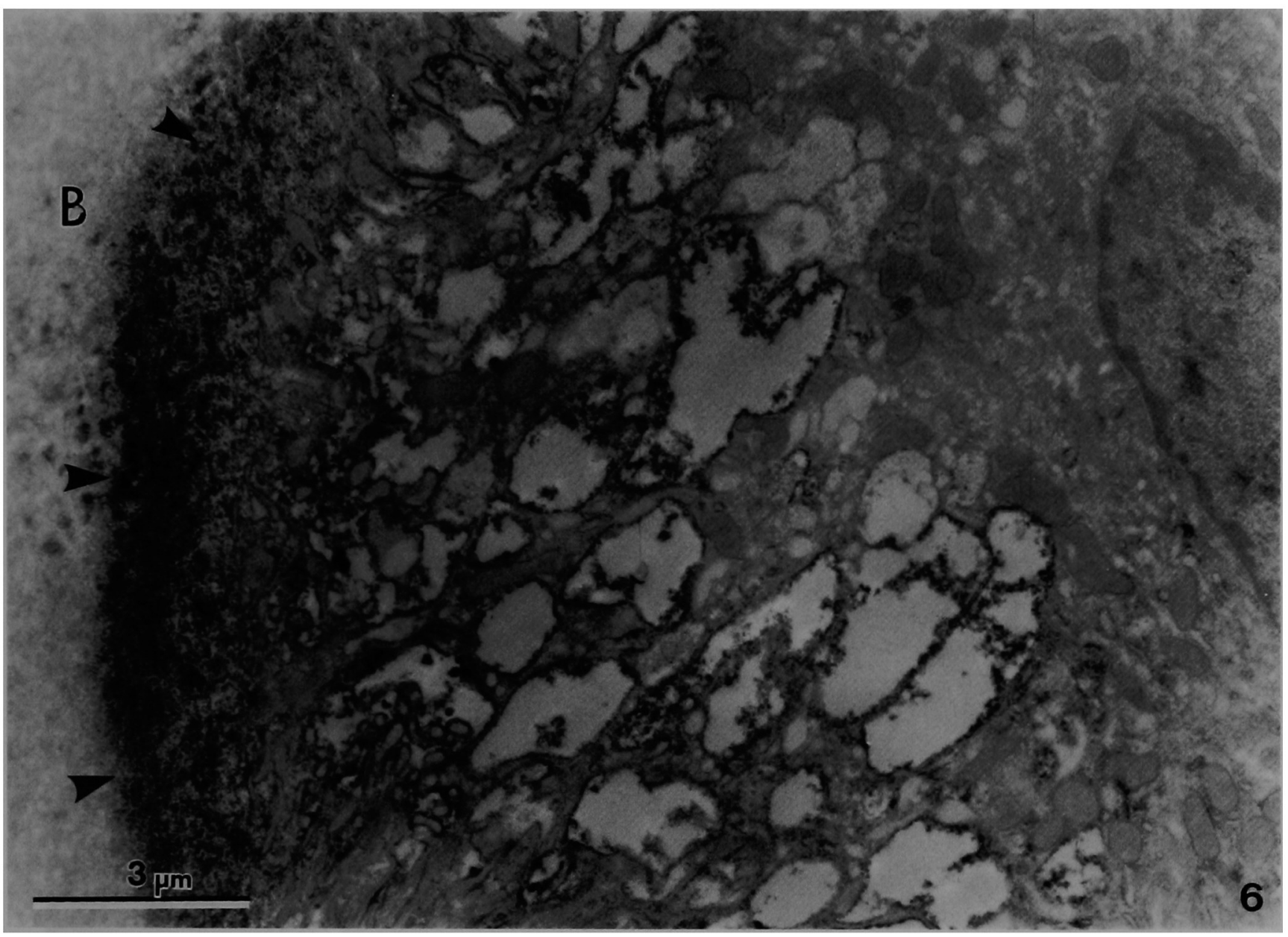

Fig. 6. An electron micrograph of a control osteoclast immunostained with the ABC method with anti cathepsin L. The immunoreaction products of cathepsin $\mathrm{L}$ are extracellularly and exclusively deposited along the resorption lacuna (arrowheads) under the ruffled borders, on the external surface of the cytoplasmic processes of the ruffled borders and at the luminal side of the ampullar dilations at the base of the ruffled borders, which appear in the section as vacuoles. Bone $(B)$. Not counterstained. $\times 10,000$.

E-64 increases to a maximum in the liver lysosomes at $6 \mathrm{hr}$. Delaissé et al. [6] also demonstrated that hydroxyproline as a product of collagen degradation by cysteine proteinases falls to a minimum level $3 \mathrm{hr}$ after the administration of E-64 in urinary excretion and then continue to $6 \mathrm{hr}$. This indicates that the degradation of collagen is inhibited by E-64 from 3 to $6 \mathrm{hr}$ after administration. Therefore, we choose $6 \mathrm{hr}$ after the injection of E-64 as the optimal time to use E-64.

Hashida et al. [17] reported that $0.5 \mathrm{mg} \mathrm{E-64/100 \textrm {g }}$ b.w. markedly inhibit cathepsin B activity but higher doses of E-64 do not show any further inhibition. When $8 \mathrm{mg}$ $\mathrm{E}-64 / 100 \mathrm{~g}$ b.w. was injected in rats, various morphological changes, as described above, were observed in the osteoclasts. However, when E-64 was reduced to 1 or $2 \mathrm{mg} / 100 \mathrm{~g} \mathrm{~b}$.w., which almost exactly corresponds to the dose ( $2 \mathrm{mg} / \mathrm{rat} 80-90 \mathrm{~g}$ b.w.) of Delaissé et al. [6], the endosome-lysosomal system appeared to be well-developed, and the other organelles showed an normal appearance and were similar to those in the normal osteoclasts described by Holtrop and King [20]. Therefore, $1 \mathrm{mg}$ (or $2 \mathrm{mg}$ ) of E-64 is considered to be the optimal dose for osteoclasts.

In the E-64-treated osteoclasts, the extracellular deposition of cathepsin $\mathrm{L}$ was suppressed, and cathepsin $\mathrm{L}$ was stored intracellularly. In the findings of Delaissé et $a l .[5,6]$ as to a inhibition of bone resorption by inhibitors using mouse calvaria cultured in medium with antipain, Tos-Lys- $\mathrm{CH}_{2} \mathrm{Cl}$ (TLCK), leupeptin [5] or E-64 [11, 19], the release of $\beta$-glucuronidase as a lysosome enzyme is suppressed in only antipain or TLCK without parathyroid hormone (PTH). Unfortunately, in the case of leupeptin or E-64, the release of $\beta$-glucuronidase was not examined under PTH free conditions, because the medium always contains PTH in addition to leupeptin or E-64. PTH induces a high degree of enzyme release, therefore the suppression of the enzyme release by E-64 might thus be weaker than stimulation by $\mathrm{PTH}$, and as a result the 


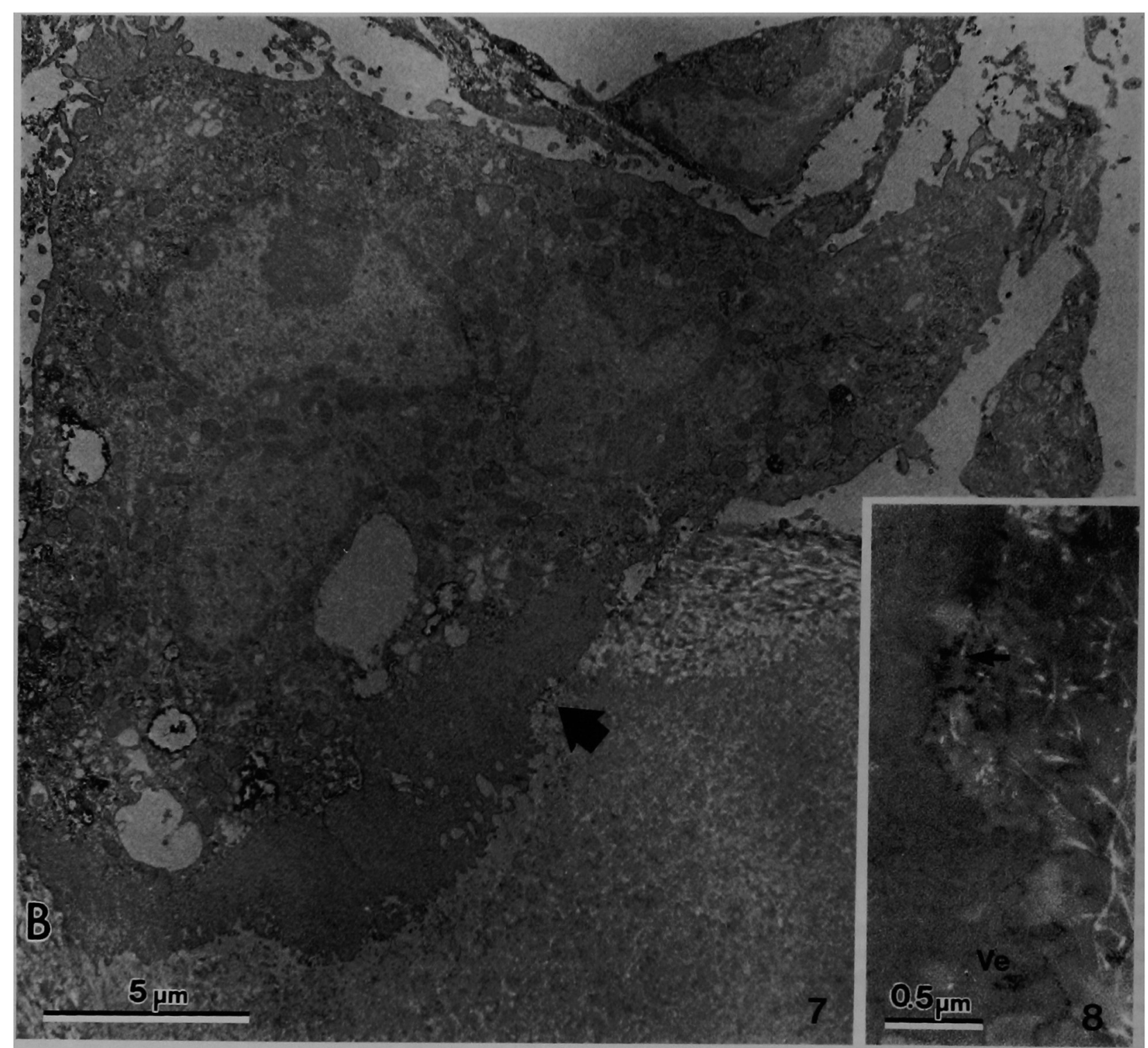

Figs. 7 \& 8. An electron micrograph of an E-64 (1 mg)-treated osteoclast immunostained with the ABC method with anti-cathepsin L. Numerous endosome-lysosomal vacuoles are well-developed in the cell. Intracellular immunoreactive products of cathepsin $L$ are found in the endosome-lysosomal vacuoles. In Fig. 8 (a higher magnification of the area indicated by the arrow in Fig. 7), only a small amount of extracelluar deposition (arrow) is found along the resorption lacuna. Not counterstained. Bone (B), cathepsin L-positive vesicle (Ve). $\times 6,000$ (Fig. 7), $\times 27,000$ (Fig. 8).

enzyme is easily released. In addition, in the other cells (except for osteoclasts) treated with cysteine proteinase inhibitors, the lysosomes become bigger than those in the normal liver cells [33], while cathepsin B and other enzymes accumulate in the bigger lysosomes $[23,26]$. Thus, judging from these findings and ours, it is certain that E-64 suppresses the release of cathepsin $L$ into the resorption lacunae. The mechanism by which E-64 suppresses the release of cathepsin $\mathrm{L}$ remains unclear but is assumed to be as follows; Nishimura et al. [29] suggested that the proteolytic conversion from procathepsin $\mathrm{L}$ to the cathepsin $\mathrm{L}$ begins in the endosomal vesicles prior to delivery to the lysosomes, and thus demonstrated that this proteolytic cleavage is blocked by either leupeptin or E-64 and is then stored as procathepsin $L$ in the endosome-lysosomal system. The proteolytic conversion may be essential for the extracellular release of cathepsin L. However, the proteolytic cleavage is not always blocked perfectly by the inhibitors, because, even in the leupeptin or E-64-treated cells, only a small amount of the processed mature enzyme is discernible. This is supported by the above findings that, even in the E-64-treated osteoclasts, small amounts of cathepsin $\mathrm{L}$ were also released in the resorption lacunae.

Lucht [24] and Tanaka and Tanaka [32] reported that osteoclasts endocytose protein (horseradish peroxidase) and peptide (microperoxidase) into the vacuoles close to 


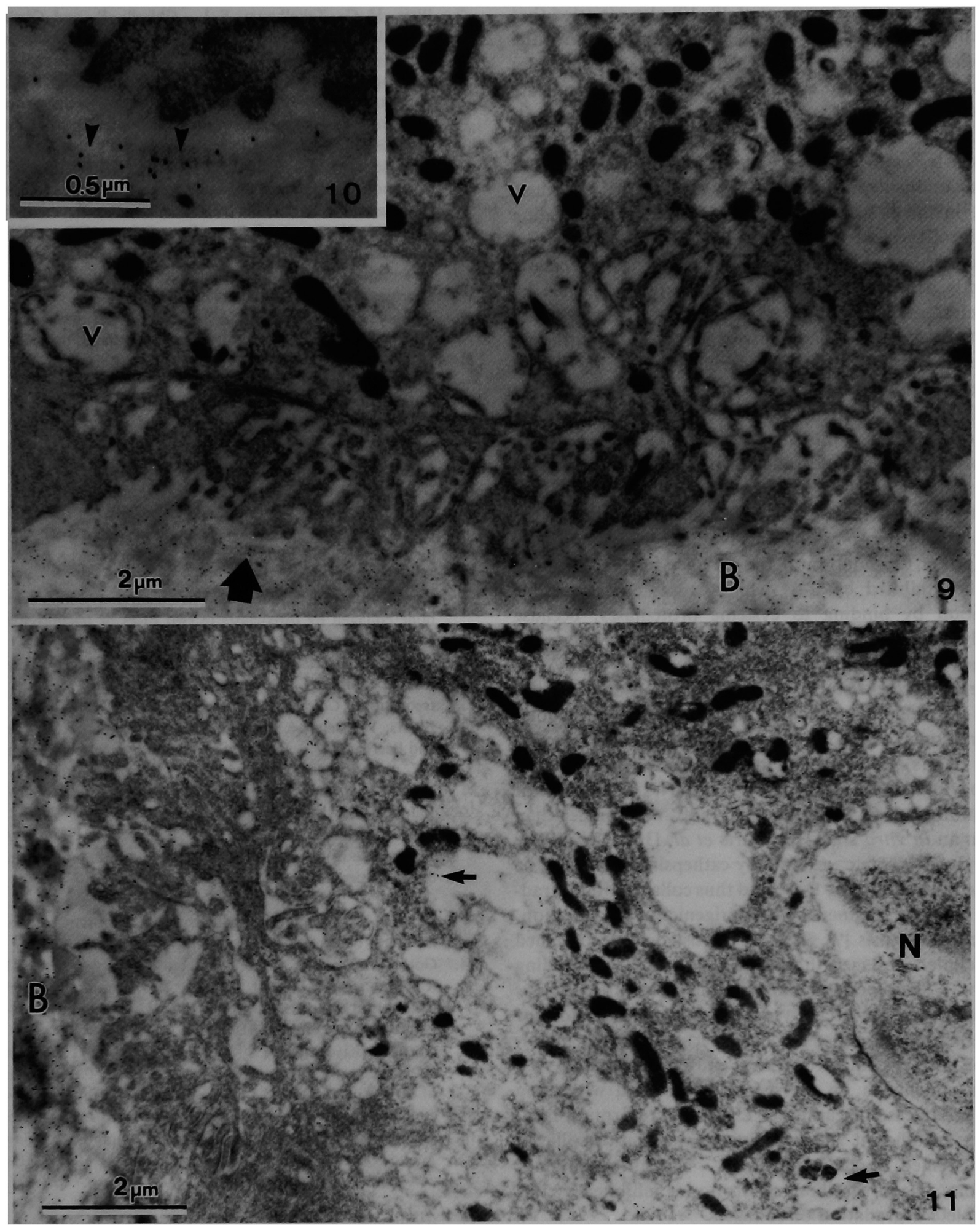

Figs. $9 \&$ 10. Electron micrographs of a control osteoclast immunostained with the immunogold method with anti-type I collagen. In Fig. 9, numerous gold particles indicating type I collagen are found on the bone matrix under the ruffied border but are not intracellularly detected in the vacuoles $(V)$. Fig. 10 is a higher magnification of the area indicated by the arrow in Fig. 9. Some typical banded collagen fibrils, such as type I collagen, are labeled with gold particles (arrowheads). Uranyl acetate staining. Bone $(B) . \times 16,000$ (Fig. 9), $\times 45,000$ (Fig. 10).

Fig. 11. An electron micrograph of an E-64 (1 mg)-treated osteoclast immunostained with the immunogold method with anti-type I collagen. Numerous gold particles indicating type I collagen are found on the bone matrix under the ruffled border in the same manner as that observed in the control osteoclasts. Moreover, the gold particles (arrows) are detected between the cytoplasmic processes of the ruffled borders, as well as in the large vacuoles, the small vacuoles, the vesicles close to the ruffled borders, and the vesicles (lysosomes) close to the nucleus $(N)$. Uranyl acetate staining. Bone $(B) . \times 13,000$. 
the ruffled borders. Everts et al. $[9,10]$ also reported that in pycnodysostosis or E-64 treated osteoclasts, some of the osteoclasts endocytose collagen fibrils into the vacuoles (lysosome-like structures). These findings thus indicate the potential capacity of endocytosis in the osteoclasts.

Goto et al. $[14,15]$ demonstrated that in normal osteoclasts, cathepsin $\mathrm{L}$ is released into the resorption lacunae and is deposited on the collagen fibrils. Furthermore, in the present study, no gold particles indicating type I collagen were found in the vacuoles close to the ruffled borders. These findings suggest that osteoclasts perfectly degrade collagen in the resorption lacunae by the released cathepsin L. On the other hand, in the E-64 treated cells, the amounts of extracellular cathepsin L was very small, and gold particles indicating type I collagen were found in the vacuoles and lysosomes. It is therefore considered that because of the insufficient release of cathepsin $\mathrm{L}$ into the resorption lacunae, collagen fibrils were partially degraded, while collagen fragments with antigenicity were formed in this lacunae. These fragments were then endocytosed and detected in the vacuoles and lysosomes by anti-type I collagen antibody. The further degradation of the collagen in the lysosomes may be inhibited by E-64 which causes an irreversible inhibition by binding to cysteine proteinases, because even lysosomes close to the nucleus still contained type I collagen with antigenicity (see Fig. 11).

In the present study, we could not find any collagen fibrils showing a periodic banding pattern in the vacuoles and lysosomes. In contrast, in cultured explants using E64 by Everts et al. [10], 4\% of the cells exhibits collagen phagocytosis under the influence of E-64. The degree of inhibition by E-64 in the present study was smaller than that in an in vitro study by Everts et al. [10]. It is likely that cysteine proteinases (mainly cathepsin L) may not be sufficiently inhibited by E-64, and thus collagen was degraded to collagen fragments with antigenicity. In addition, in pycnodysostosis [9], 92\% of the osteoclasts contained intracellular collagen fibrils with a periodic banding pattern. Their possible explanation for this is perhaps the fact that in pycnodysostosis, the degradation of collagen fibrils is almost blocked perfectly by the inhibition of both cysteine proteinases and collagenase.

In conclusion, in the osteoclasts treated with $1 \mathrm{mg}$ E-64, the release of cathepsin $\mathrm{L}$ into the resorption lacunae was insufficient and thus the degradation of collagen fibrils seemed to be suppressed. The above findings therefore suggest that bone resorption is inhibited by this incomplete degradation of collagen.

\section{References}

1. Baron, R., Neff, L., Brown W., Courtoy, P. J., Louvard, D. and Farquhar, M. G.: Polarized secretion of lysosomal enzymes: co-distribution of cation-independent mannose-6-phosphate receptors and lysosomal enzymes along the osteoclast exocytic pathway. J. Cell Biol. 106; 1863-1872, 1988.
2. Blair, H. C., Teitelbaum, S. L., Ghiselli, R. and Gluck, S.: Osteoclastic bone resorption by polarized vacuolar proton pump. Science 245; 855-857, 1989.

3. Debari, K., Sasaki, T., Udagawa, N. and Rifkin, B. R.: An ultrastructural evaluation of the effects of cysteine-proteinase inhibitors on osteoclastic resorptive functions. Calcif. Tissue Int. 56; 566-570, 1995.

4. Delaissé, J. M. and Vaes, G.: Mechanism of mineral solubilization and matrix degradation in osteoclastic bone resorption. In "Biology and Physiology of the Osteoclast." ed. by B. R. Rifkin, C. V. Gay, CRC Press, Boca Raton, 1992, pp 289-314.

5. Delaissé, J. M., Eeckhout, Y. and Vaes, G.: Inhibition of bone resorption in culture by inhibitors of thiol proteinases. Biochem. J. 192; 365-368, 1980.

6. Delaissé, J. M., Eeckhout, Y. and Vaes, G.: In vivo and in vitro evidence for the involvement of cysteine proteinases in bone resorption. Biochem. Biophys. Res. Commun. 125; 441447, 1984.

7. Delaissé, J. M., Boyde, A., Maconnachie, E., Ali, N. N., Sear, C. H. J., Eeckhout, Y., Vaes, G. and Jones, S. J.: The effect of inhibitors of cysteine-proteinases and collagenase on the resorptive activity of isolated osteoclasts. Bone 8; 305-313, 1987.

8. Delaissé, J. M., Eeckhout, Y., Neff, L., François-Gillet, Ch., Henriet, P., Su, Y., Vaes, G. and Baron, R.: (Pro)collagenase (matrix metalloproteinase-1) is present in rodent osteoclasts and in the underlying bone resorbing compartment. J. Cell Sci. 106; 1071-1082, 1993.

9. Everts, V., Aronson, D. C. and Beertsen, W.: Phagocytosis of bone collagen by osteoclasts in two cases of pycnodysostosis. Calcif. Tissue Int. 37; 25-31, 1985.

10. Everts, V., Beertsen, W. and Reinier, S.: Effects of the proteinase inhibitors leupeptin and E-64 on osteoclastic bone resorption. Calcif. Tissue Int. 43; 172-178, 1988.

11. Everts, V., Delaissé, J. M., Korper, W., Niehof, A., Vaes, G. and Beertsen, W.: Degradation of collagen in the bone-resorbing compartment underlying the osteoclast involves both cysteine-proteinases and matrix metallo-proteinases. J. Cell Biol. 150; 221-231, 1992.

12. Fuller, K. and Cambers, T. J.: Localisation of mRNA for collagenase in osteocytic, bone surface and chondrocytic cells but not osteoclasts. J. Cell Sci. 108; 2221-2230, 1995.

13. Goto, T., Tsukuba, T., Ayasaka, N., Yamamoto, K. and Tanaka, T.: Immunocytochemical localization of cathepsin D in the rat osteoclast. Histochemistry 97; 13-18, 1992.

14. Goto, T., Tsukuba, T., Kiyoshima, T., Nishimura, Y., Kato, K., Yamamoto, K. and Tanaka, T.: Immunohistochemical localization of cathepsin B, D and $\mathrm{L}$ in the rat osteoclast. Histochemistry 99; 411-414, 1993.

15. Goto, T., Kiyoshima, T., Moroi, R., Tsukuba, T., Nishimura, Y., Himeno, M., Yamamoto, K. and Tanaka, T.: Localization of cathepsins $\mathrm{B}, \mathrm{D}$, and $\mathrm{L}$ in the rat osteoclast by immuno-light and -electron microscopy. Histochemistry 101; 33-40, 1994.

16. Hanada, K., Tamai, M., Yamagishi, M., Ohmura, S., Sawada, J. and Tanaka, I.: Isolation and characterization of E-64, a new thiol proteinase inhibitor. Agric. Biol. Chem. 42; 523528, 1978.

17. Hashida, S., Kominami, E. and Katunuma, N.: Inhibition of cathepsin B and cathepsin L by E-64 in vivo. II. Incorporation of $\left[{ }^{3} \mathrm{H}\right] \mathrm{E}-64$ into rat liver lysosomes in vivo. J. Biochem. 91; 1373-1380, 1982.

18. Hashida, S., Towatari, T., Kominami, E. and Katunuma, N.: Inhibitions by E-64 derivatives of rat liver cathepsin B and cathepsin $\mathrm{L}$ in vitro and in vivo. J. Biochem. 88; 1805-1811, 1980.

19. Hill, P. A., Buttle, D. J., Jones, S. J., Boyde, A., Murata, M., Reynolds, J. J. and Meikle, M. C.: Inhibition of bone resorp- 
tion by selective inactivators of cysteine proteinases. J. Cell. Biochem. 56; 118-130, 1994.

20. Holtrop, M. E. and King, G. J.: The ultrastructure of the osteoclast and its functional implications. Clinical Orthopaedics and Related Research 123; 177-196, 1977.

21. Kakegawa, H., Nikawa, T., Tagami, K., Kamioka, H., Sumitani, K., Kawata, T., Drobnic-Kosorok, M., Lenarcic, B., Turk, V. and Katunuma, N.: Participation of cathepsin L on bone resorption. FEBS Lett. 321; 247-250, 1993.

22. Kirschke, H., Kembhavi, A. A., Bohley, P. and Barrett, A. J.: Action of rat liver cathepsin $L$ on collagen and other substrates. Biochem. J. 201; 367-372, 1982.

23. Kopitz, J., Kisen, G. Ø., Gordon, P. B., Bohley, P. and Seglen, P. O.: Nonselective autophagy of cytosolic enzymes by isolated rat hepatocytes. J. Cell Biol. 111; 941-953, 1990.

24. Lucht, U.: Absorption of peroxidase by osteoclasts demonstrated by electron microscopic histochemistry. Histochemie 29; 274-286, 1972.

25. Maciewicz, R. A., Etherington, D. J., Kos, J. and Turk, V.: Collagenolytic cathepsins of rabbit spleen: a kinetic analysis of collagen degradation and inhibition by chicken cystatin. Coll. Relat. Res. 7; 295-304, 1987.

26. Matsui, K., Shirasawa, N. and Eto, Y.: Cytoplasmic accumulations in rat primary brain cell cultures following treatment with E-64, a thiol protease inhibitor. Dev. Neurosci. 12; 133-139, 1990.

27. Ngyuen, Q., Mort, J.S. and Roughley, P. J.: Cartilage proteoglycan aggregate is degraded more extensively by cathepsin $\mathrm{L}$ than by cathepsin B. Biochem. J. 266; 569-573, 1990.
28. Nishimura, Y., Kawabata, T., Yano, S. and Kato, K.: Inhibition of intracellular sorting and processing of lysosomal cathep$\sin \mathrm{H}$ and $\mathrm{L}$ at reduced temperature in primary cultures of rat hepatocytes. Arch. Biochem. Biophys. 283; 458-463, 1990.

29. Nishimura, Y., Kato, K., Furuno, K. and Himeno, M.: Inhibitory effect of leupeptin on the intracellular maturation of lysosomal cathepsin $\mathrm{L}$ in primary cultures of rat hepatocytes. Biol. Pharm. Bull. 18; 945-950, 1995.

30. Rifkin, B. R., Vernillo, A. T. and Kleckner, A. P.: Cathepsin $B$ and $\mathbf{L}$ activities in isolated osteoclasts. Biochem. Biophys. Res. Commun. 179; 63-69, 1991.

31. Takita, K., Ohsaki, Y., Nakata, M. and Kurisu, K.: Immunofluorescence localization of type I and type III collagen and fibronectin in mouse dental tissues in late development and during molar eruption. Archs. oral Biol. 32; 273-279, 1987.

32. Tanaka, T. and Tanaka, M.: Cytological and functional studies of preosteoclasts and osteoclasts in the alveolar bones from neonatal rats using microperoxidase as a tracer. Calcif. Tissue Int. 42; 267-272, 1988.

33. Tanaka, K., Ikegaki, N. and Ichihara, A.: Purification and characterization of hemoglobin-hydrolyzing acidic thiol protease induced by leupeptin in rat liver. J. Biol. Chem. 259; 5937-5944, 1984.

34. Väänänen, H.K., Karhukorpi, E. K., Sundquist, K., Wallmark, B., Roininen, I., Hentunen, T., Tuukkanen, J. and Lakkakorpi, P.: Evidence for the presence of proton pump of the vacuolar $\mathrm{H}^{+}$-ATPase type in the ruffled borders of osteoclasts. J. Cell Biol. 111; 1305-1311, 1990. 